\title{
THEORETICAL CHARACTERIZATION OF TRANSITION STATE DYNAMICAL RESONANCES IN HEAVY-LIGHT-HEAVY REACTIONS
}

\author{
A. AGUILAR, M. ALBERTÍ, R. BLASCO, M. GILIBERT, \\ X. GIMÉNEZ, M. GONZÁLEZ, J. M. LUCAS, M. PRIETO, \\ R. SAYÓS and A. SOLÉ \\ Departament de Química Física, Universitat de Barcelona Martí i Franquès 1 \\ 08028 Barcelona (Spain)
}

\begin{abstract}
The resonant reactivity of three elementary Heavy-Light-Heavy reactions is presented and discussed. Collinear reactivity, in which a vibrational adiabatic model is constructed, is used for a detailed analysis of resonance phenomena, which appear as a direct consequence of transition state metastable states in the strong interaction region of the potential energy surface. Their influence on the detailed mechanism of the elementary process is also discussed. The shape of the resonant peak, and the phase and the Argand plot of the S-matrix are used for a further characterization.

Three-dimensional approximate calculations are used to test the evolution of the energy dependent structure present in detailed quantities when sums and integrations over all partial waves contributing to reaction are taken into account to obtain the usual averaged global quantities such as integral state-tostate cross sections.
\end{abstract}

KEY WORDS: Reaction dynamics, transition state, resonances, heavy-light-heavy mass combination.

\section{INTRODUCTION}

The characterization of the transition state (TS) region of the potential energy surface (PES) associated to a collision system constitutes one of the more challenging topics in collision and reaction dynamics. Since the early days of chemical kinetics, its fundamental role in reaction rate control has been established. However, obtaining direct information on this region is still a rather difficult task and only very recently experiments in this direction have been reported.$^{1-2}$ In addition, theoretical quantum chemistry methods are not still completely capable of providing its geometry and energetics with sufficient accuracy - which has been commonly established in $1 \mathrm{kcal} /$ mol-for systems more complicated than $\mathrm{H}+\mathrm{H}_{2}$.

For this reason, indirect methods have been used, both from experiment and theory, to test the accuracy of proposed transition state structures. In this sense, reactive state-to-state total and differential reactive cross sections have been measured and calculated for a wide variety of systems, which, together with certain types of molecular spectra, constitute an important source of information. ${ }^{2}$ 
From the experimental threshold reactivity and shape of the excitation function, the height and position of the transition state barrier can be roughly inferred and, conversely, calculated cross sections from proposed TS may lead, after comparison with experimental data, to a further improvement of the global PES and particularly of the TS. This trial and error procedure has been commonly used to optimize the topography of potential surfaces. However, dynamical tests based only on these features cannot provide accurate information on enough points of the PES, as has been demonstrated in more complete tests which show important disagreements between experiments and exact three-dimensional quantum-mechanical calculations. ${ }^{3}$

One way to obtain additional information of the PES's characteristics, and particularly of the TS region, emerges from the detailed study of the resonant reactivity. Resonances, first discovered and characterized in fundamental particle physics, have been reported in reaction dynamics twenty years ago from theoretical calculations of collinear atom-diatom reactive collisions. ${ }^{4}$ They can be described as sharp reactivity peaks or wells superimposed over a smooth-varying background reactivity as a function of total or relative collision energy. They seem to be of purely quantum nature, although certain controversy exists on this point since classical analogs to resonances have been established: the Resonant Periodic Orbits (RPO), ${ }^{5}$ which are described as trapped or quasi-trapped quasiclassical trajectories around the TS region. However, the sharpness and energy-especificity of the quantum peaks have not been reproduced by the existing calculations on RPO's. ${ }^{6}$

It is precisely these latter features that make resonances suitable as a fine-tuned tool for probing potential energy surfaces. The magnitude and position of the resonant peaks are greatly influenced by the curvature of the modes perpendicular to the reaction path in the TS region. For this reason, inversion of precise information on the potential should be possible. ${ }^{2}$

Resonances also influence greatly the reactive dynamical behaviour of a collision system. They are a direct consequence of the presence of metastable states in the PES, and can be justified from a vibrationally adiabatic picture of the reactive process. Within this scheme, the existence of more relaxed vibrational modes in the TS region, when compared to the asymptotes, allow the presence of vibrationally adiabatic wells which may support bound or quasi-bound discrete energy levels. Thus, resonances occur when the system energy remains trapped in those discrete levels, while direct reaction occurs when the system energy concentrates on the reactive non-discretized mode.

It is our purpose in this article to provide new data about the resonant behaviour of three Heavy-Light-Heavy (HLH) reactions:

$$
\begin{aligned}
\mathrm{C}+\mathrm{HF} & \rightarrow \mathrm{CH}+\mathrm{F} \\
\mathrm{C}+\mathrm{HI} & \rightarrow \mathrm{CH}+\mathrm{I} \\
\mathrm{Cl}+\mathrm{HCl} & \rightarrow \mathrm{ClH}+\mathrm{Cl}
\end{aligned}
$$

The first two reactions are studied within the collinear approach. ${ }^{7}$ Although this restriction neglects the contribution from the orbital and rotational angular 
momenta to reactivity, the feasibility of the calculations enable a very fine-grid scanning over the energy scale which makes a detailed analysis of each resonant peak possible.

The third reaction has been studied under the Reactive Infinite Order Sudden Approximation (R-IOSA) approach, ${ }^{8}$ which allows to explore the reactive process in the three physical dimensions. The sudden approximation included in this technique completely decouples the angular motion, in such a way that the reactive cross section must be obtained by integrating fixed-angle cross sections over the atom-diatom angle of approach. This procedure is specially suited to test the evolution of the reactivity from one to three dimensions, and particularly to analyze the evolution of possible structures in the excitation function when all the angular momentum partial waves for each orientation angle are taken into account.

In the next sections a brief description of the methods used for the calculations is made, followed by the presentation and analysis of the corresponding results. Finally, the main conclusions arising from the analysis are summarized.

\section{METHODOLOGY}

In this section, the quantum-mechanical methods used for the calculations are only briefly outlined, since they have been described with great detail elsewhere. ${ }^{7-8}$ The $\mathrm{R} 1$ and $\mathrm{R} 2$ reactions have been studied under the collinear restriction approach using Hyperspherical Coordinates (HC), which substitute the two Jacobi distances by a hyperradius and a polar angle. They have been shown as the best coordinate framework to deal with the strong reaction-path curvature characteristic of HLH systems, and provide also an alternative way to understand the reactive process. Particularly, the quasi-separability of the hyperradius coordinate from the scattering equations intuitively allows the introduction of an adiabatic approximation for the angular motion. Although the angular coordinate associated involves the two reaction channels as a whole, the analysis of the wave function density probability indicates that its value is concentrated only on one channel when the hyperradius reaches asymptotic values, and spreads over the whole angular range in the strong interaction region. ${ }^{9}$ This fact allows to identify the angular motion with the vibration perpendicular to the reaction mode. For this reason, the adiabatic approximation is known as the DIVAH model ${ }^{9}$ (Diagonally corrected Vibrational Adiabatic Hyperspherical model) and makes it possible to construct Vibrational Adiabatic (VA) curves obtained from the evolution of the vibrational levels along the hyperradius. These VA curves will constitute the basis for the interpretation of the resonant collinear reactivity for the first two reactions.

The R3 reaction has been studied using the R-IOSA method, which treats the reactive process in the physical three dimensions but in an approximate way. The Infinite Order Sudden Approximation considers the translation of the incoming atom to be very fast as compared with the rotational motion of the target molecule, in such a way that the atom-diatom orientation angle can be kept fixed during the collision process. This leads to a complete decoupling of the angular motion in the 
scattering equations, so that a different set of coupled two-mathematicaldimensional (2MD) differential equations is solved for each orientation angle. The fixed-angle cross sections thus derived-obtained after summing over all the orbital angular momentum partial waves-are then integrated over the orientation angle to obtain the total state-to-state reactive cross sections:

$$
\sigma_{v_{\alpha} j_{\alpha}} \longrightarrow v_{\lambda}^{\prime} \text { all } j_{\lambda}^{\prime}=\frac{\pi}{k_{v_{\alpha} j_{\alpha}}^{-2}} \sum_{l=0}^{\infty}(2 l+1) \frac{1}{2} \int_{-1}^{1}\left|S_{v_{\alpha}}^{l} \longrightarrow v_{\lambda}\left(\gamma_{\alpha}\right)\right|^{2} d \cos \gamma_{\alpha}
$$

From the analysis of the energy dependent structure of detailed quantities such as single- $l$ contributions to fixed-angle cross sections and its evolution once sums and integrations are performed, to obtain averaged quantities such as the total cross section, the "history" of possible structures can be followed, thus providing a route to determine the maintenance of resonances when global quantities are considered.

\section{DESCRIPTION AND ANALYSIS OF RESULTS}

\section{Collinear Results}

Background reactivity of collinear reactions $\mathrm{R} 1$ and $\mathrm{R} 2$ has been described with detail elsewhere ${ }^{10}$ so we will center here directly on resonance features. The energy dependence of the reaction probability for several transitions is shown in Figures 1 and 2 for reactions R1 and R2, respectively. In Figure 1, the VA curves for the C + $\mathrm{HF}$ system have been incorporated to enable a direct comparison between resonant peaks and bound levels, while the VA curves for the C + HI system are shown in Figure 3. Reaction R1, highly endothermic, is characterized by the presence of several peaks which may be identified as resonances. On the contrary, reaction R2 presents higher background reactivity, as we can expect from a slightly exothermic reaction, and a part of the resonant contribution is possibly masked. However, the most important peaks are still present.

In spite of its shortcomings, the collinear restriction approach has become newly actualized in view of recent experiments ${ }^{2}$ on the photodetachment spectra of stable linear triatomic anions which directly probe the collinear saddle region of the neutral dissociating system.

The identification of a reactivity peak as a resonance can be made in several ways. From formal scattering theory, resonances are defined as poles of the scattering matrix in the complex linear momentum plane (with negative contribution to the pure imaginary part) or in the complex energy plane (with positive real part and negative imaginary part). ${ }^{11}$ Throughout a resonance, the phase of the scattering matrix is expected to suddenly increase its value in an amount of approximately $\pi$. The contribution of the background reactivity and the distance of the pole to the real axis tend to decrease the magnitude of this sharp variation.

The interference between the background and resonant reactivities also determine the global shape of the peak. Thus, an increasing background contribution causes the appearance of oscillations instead of pure maxima or minima. Finally, resonances 

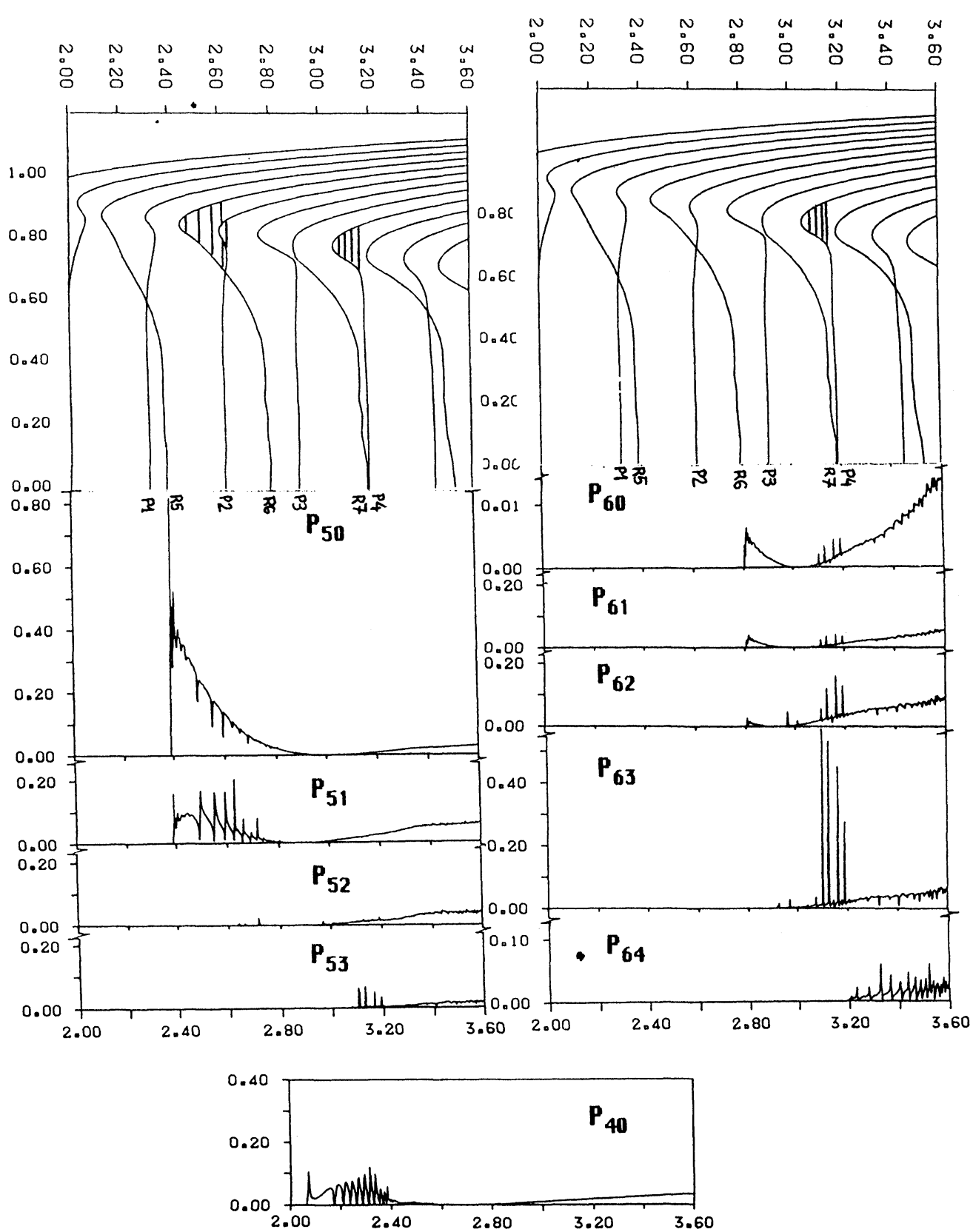

Figure 1 Reaction probabilities for the $\mathrm{C}+\mathrm{HF}$ system as a function of relative collision energy (in $\mathrm{eV}$ ) for different $v \rightarrow v^{\prime}$ transitions (identified as $P_{v v^{\prime}}$ ). In the upper part, the Vibrational Adiabatic curves are included for comparison between peak positions and the energy of bound or quasi-bound levels. The asymptotic correlation with reactant and product vibrational diatomic levels is also shown (as $\mathbf{R v}$ or $\mathbf{P v}^{\prime}$, respectively). 


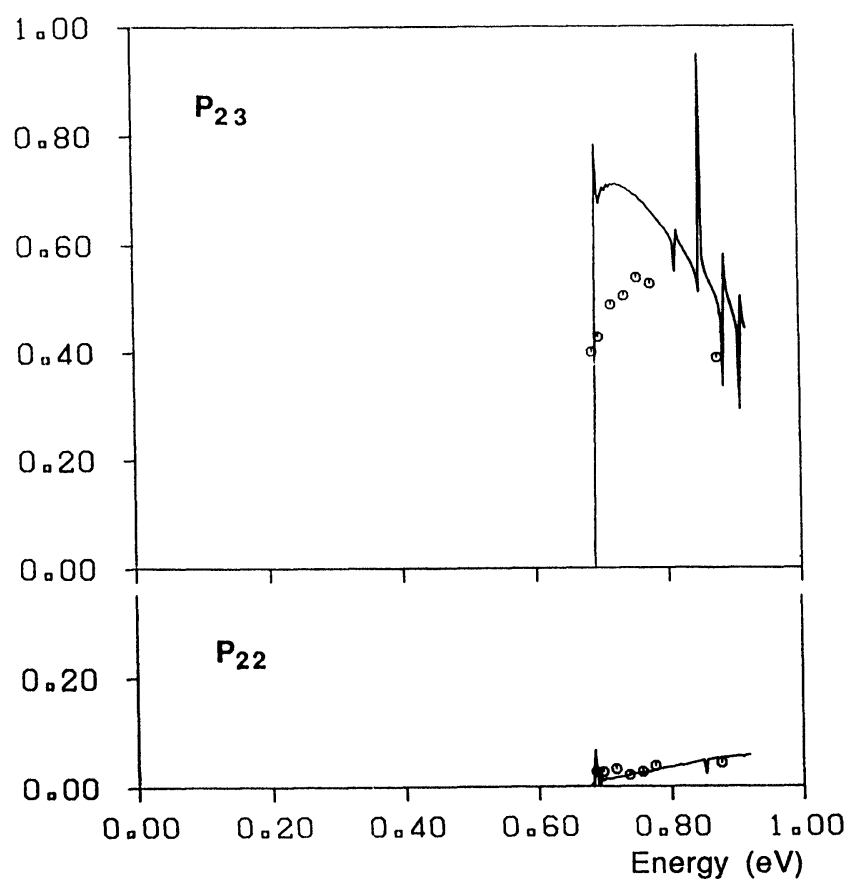

Figure 2 Reaction probabilities as a function of relative collision energy for the C $+\mathrm{HI}$ system. Solid line: Quantum results. circles: Quasiclassical results.

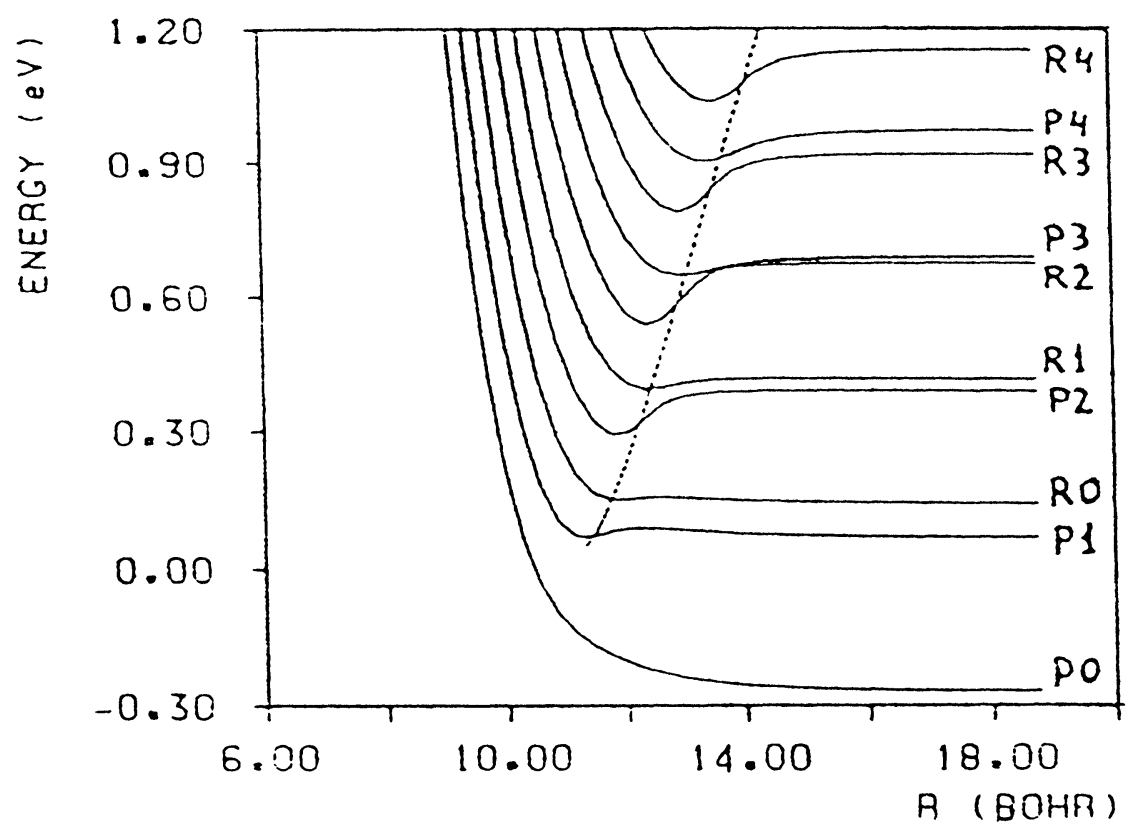

Figure 3 Vibrational Adiabatic curves for the $\mathrm{C}+\mathrm{HI}$ system. The dotted line represents the potential ridge, the maximum between the two reactant and product valley minima. Asymptotic vibrational levels shown as in Figure 1. 


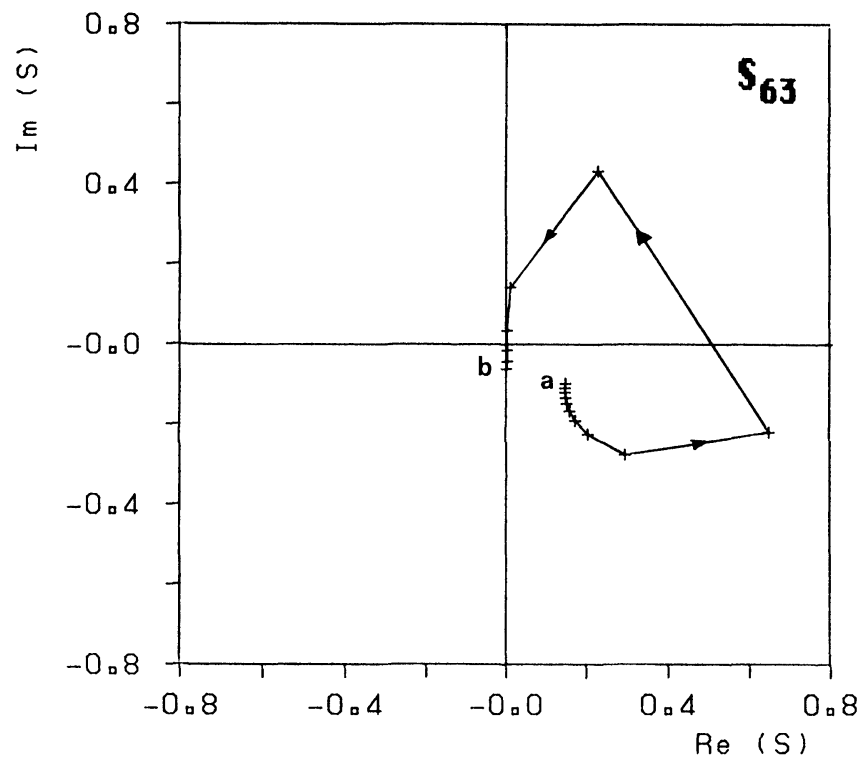

a : 3.1620 eV

b : $3.1650 \mathrm{eV}$

step : 0.0002 eV

Figure 4 Phase of the S-matrix as a function of total energy for the $6 \rightarrow 3$ transition. C + HF system. At the resonance energy position, a sudden variation of approx. 140 degrees is encountered.

can also be identified by plotting the real part of the scattering matrix versus the imaginary part, at different values of the total energy-Argand plots. In the vicinity of a resonance, a counter-clockwise variation is expected to be encountered, the magnitude of the resonance being proportional to the radius of the circle thus obtained. All these features are shown in Figures 4-6 for reaction R1.

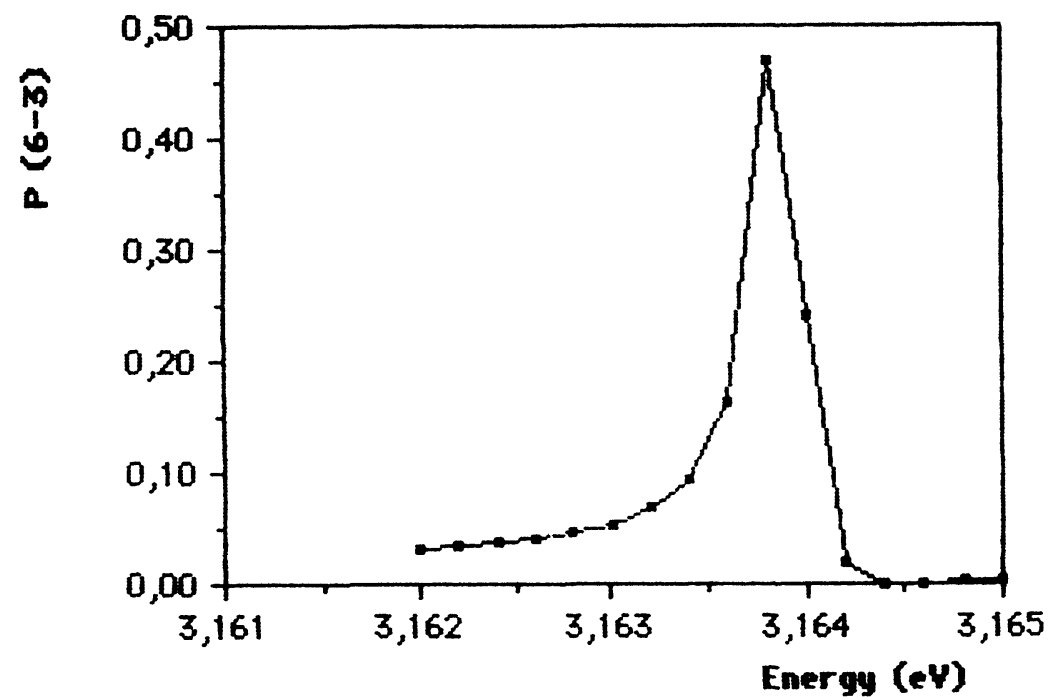

Figure 5 Shape of a resonant peak for the $6 \rightarrow 3$ transition. C + HF system. The deviation from the pure Breit-Wigner form is due to the interference between direct and resonant scattering. 


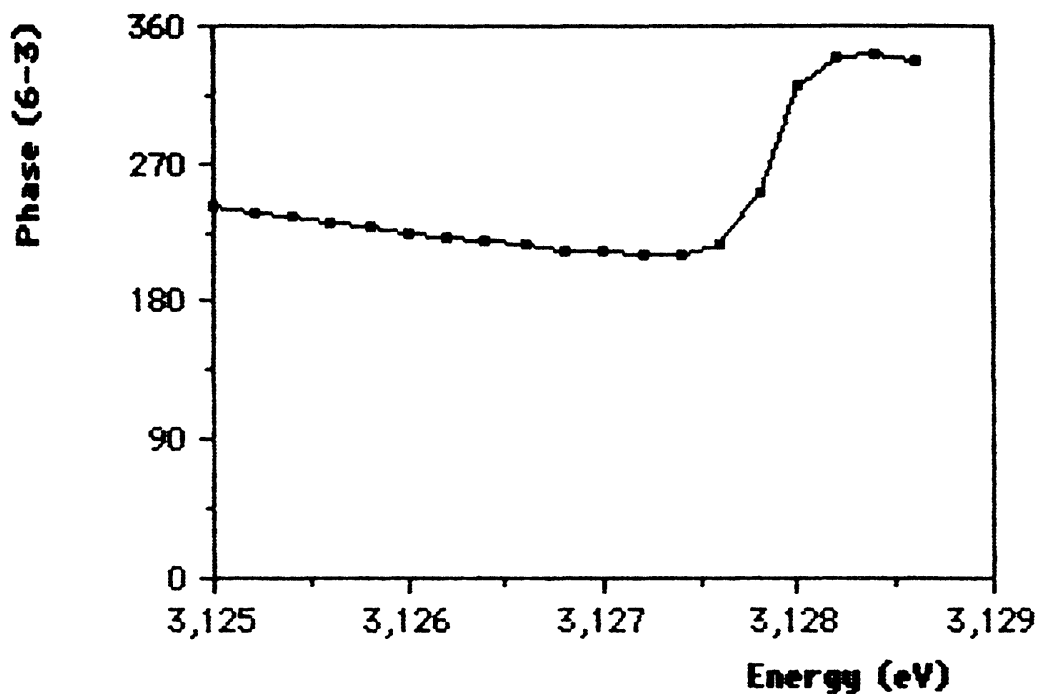

Figure 6 Argand plot for the $6 \rightarrow 3$ transition. C + HF system. A clear circular evolution should be obtained with higher energy resolution.

The VA curves of Figures 1 and 3 constitute a simple and intuitive model that can be used to understand and justify the origin of the resonant peaks. The discrete energy levels emerging from the wells supported by the VA curves correlate directly with the maxima or minima of the reaction probability when represented versus the collision energy. At energies corresponding to saddle bound or quasi-bound levels the system energy remains stored in the perpendicular vibrational mode, while above and below any discrete level the system energy is concentrated in the reactive mode. This fact serves as a qualitative justification of the observed probability profiles: smooth-varying background reactivity with superimposed pronounced peaks.

The vibrational adiabaticity condition specially holds for HLH systems, ${ }^{12}$ thus providing a qualitative tool for understanding the general dynamics of this kind of systems. However, this condition is not fulfilled completely during the whole reactive process, and especially when resonances dominate the reaction. Thus, series of resonances appear at energy positions independent of the particular $v \rightarrow v^{\prime}$ transition considered. In this case, the correlating bound or quasi-bound levels act as intermediates in the detailed mechanism of the reaction. The region where the adiabaticity is broken corresponds to the dotted line in the VA curves (Figure 3), the so-called potential ridge.

\section{Three-Dimensional Results}

Results of an extended R-IOSA calculation for the R3 reaction are presented in Figures 7-9. Details of the general dynamic behaviour will be given elsewhere. ${ }^{13}$ From Figure 7 it is particularly worth noticing the presence of a pronounced peak at high $l$ values, which moves forward as total energy increases. 


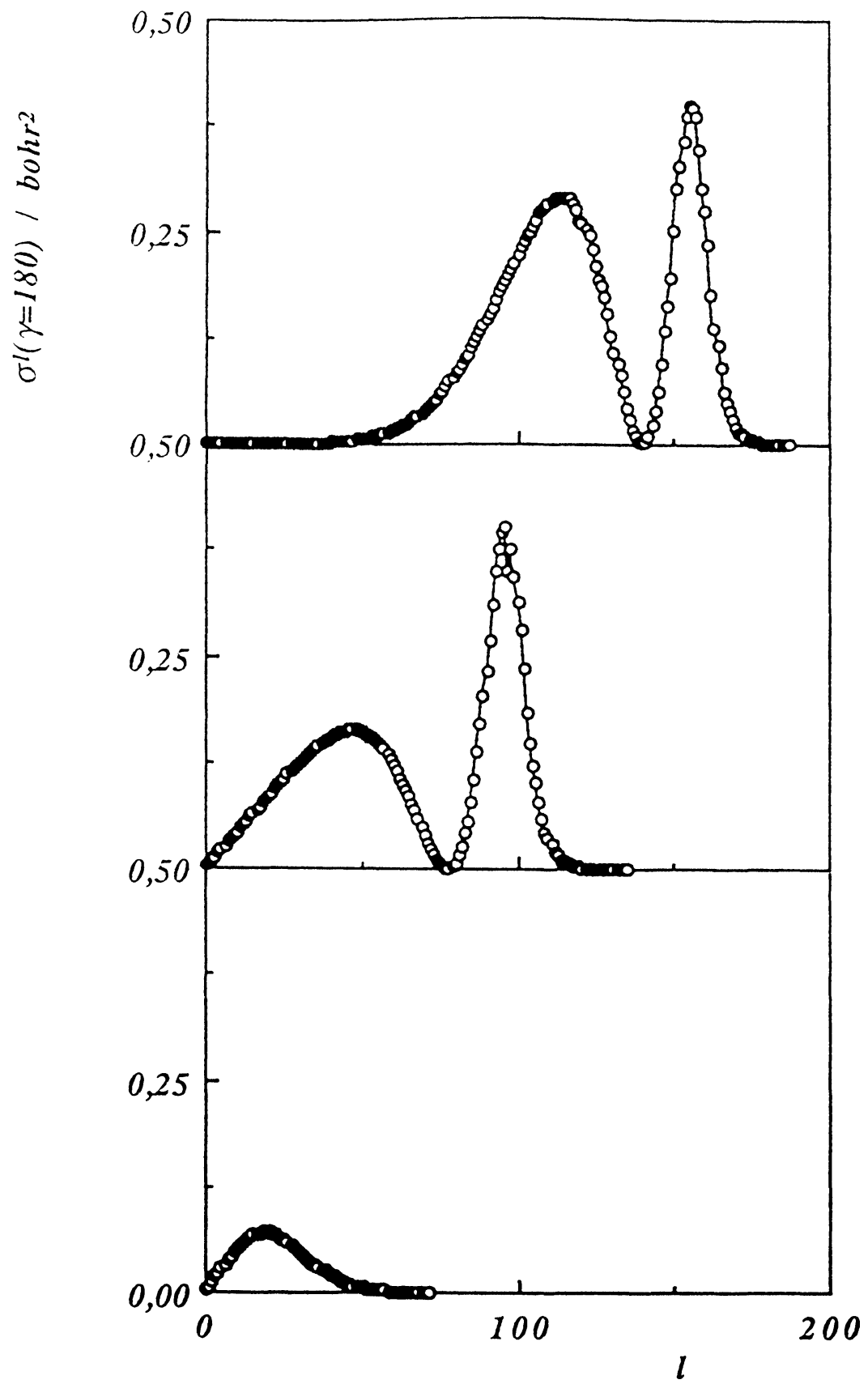

Figure $7 \mathrm{Cl}+\mathrm{HCl}$ single $l$ (orbital angular momentum) contributions to fixed angle cross sections as a function of $l$ at 180 degree $\left(\gamma\right.$, orientation angle) and $E_{\text {tot }}=0.37$ (lower panel), 0.50 (central) and 0.70 (upper) eV. Transition $v=0 \rightarrow$ all $v^{\prime}$. 


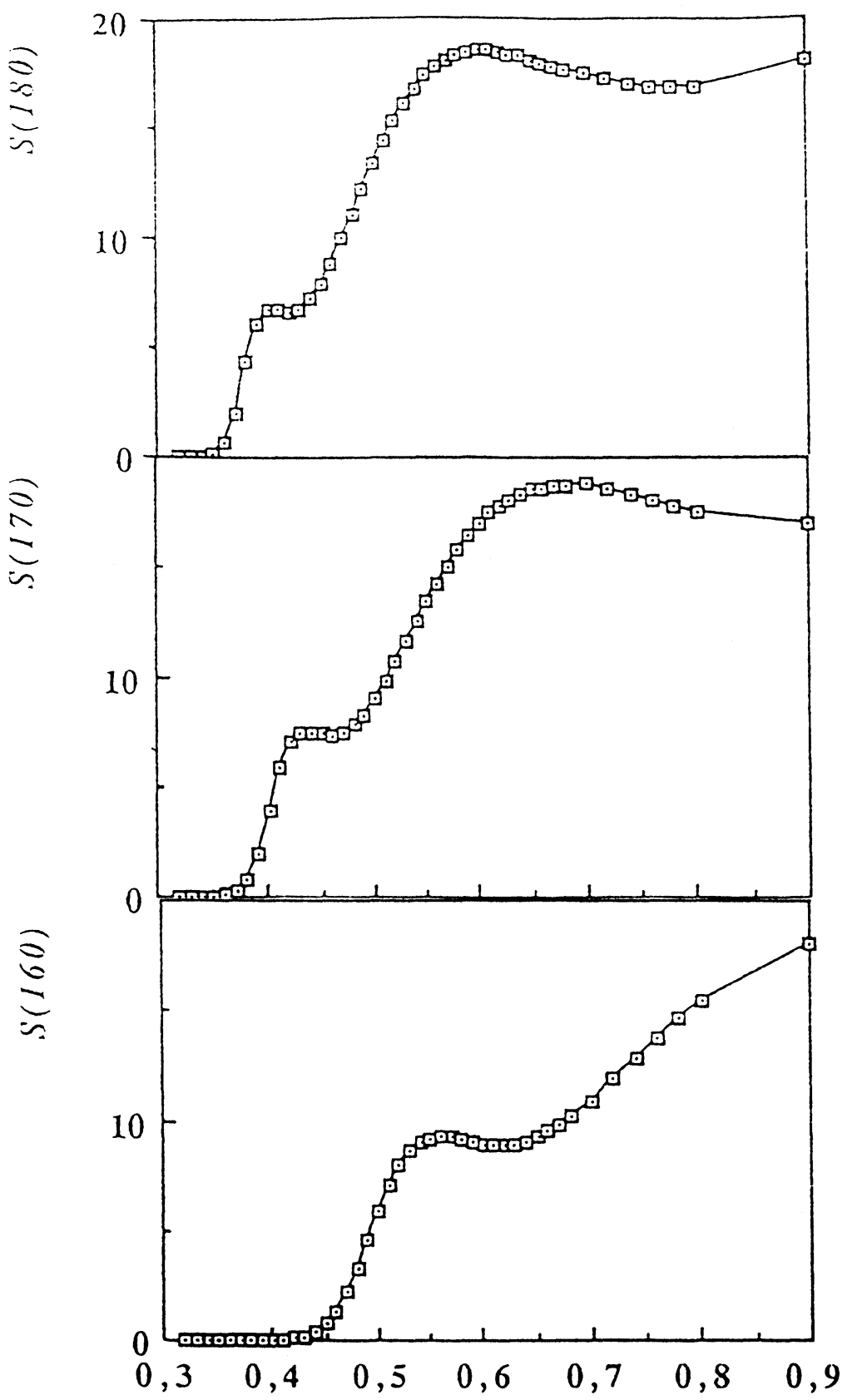

Figure $8 \mathrm{Cl}+\mathrm{HCl}$ fixed angle cross section as a function of total energy $(\mathrm{eV})$ for $\gamma=180,170$ and 160 degrees. Transition $v=0 \rightarrow$ all $v^{\prime}$. 


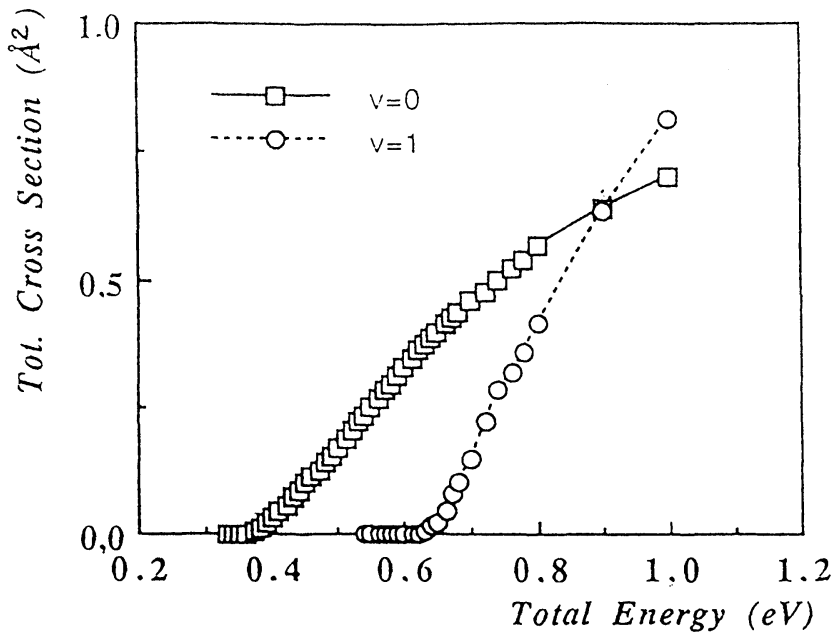

Figure $9 \mathrm{Cl}+\mathrm{HCl}$ total cross section as a function of total energy for the transitions $v=0 \rightarrow$ all $v^{\prime}$ and $v=1 \rightarrow$ all $v^{\prime}$.

A certain remaining oscillating reactivity is found in Figure 8 in the fixed angle cross sections, which are summed over the orbital angular momentum. It is maintained, although shifted to higher energies, when the approach angle is displaced gradually away from collinearity. However, a complete smoothing down of the structured behaviour is observed when the detailed quantities are summed over the angular momentum and integrated over the orientation angle (Figure 9). Miller and Zhang ${ }^{6}$ have analysed this feature in terms of broad and narrow resonances in the angular momentum space. A narrow resonance emerges when only one value of $l$ makes a contribution to the resonance structure for a given energy, so that resonance structure will be observed in the energy dependence of the integral cross section. A broad resonance is obtained when many values of $l$ contribute to the integral cross section, so that the resonance structure may be quenched by the overlapping resonances. In our case, a broad resonance is centered on different values of the orbital angular momentum for different values of the orientation angle. This results in a complete disappearance of any structure when integrations are performed.

\section{CONCLUSIONS}

In this article the resonant behaviour of three HLH reactions has been presented and analysed, both from collinear and approximate three-dimensional approaches. The collinear reactivity allows a detailed analysis of the resonant peaks, where the shape of the peak, the phase and Argand plots of the S-matrix serve as complementary tools to their characterization. The important influence in the detailed mechanism of the reactive collision can also be inferred from a vibrational adiabatic picture of the process, in the sense that the existence of a discrete level in a given VA curve causes the appearance of a resonance at the corresponding energy value for transitions in 
which this VA curve is not involved (the VA curve does not correlate asymptotically with any of the vibrational levels for the transition considered).

The approximated three-dimensional study serves as a first criterion to test the evolution of existing structures in detailed quantities when sums and integrations are performed to obtain averaged quantities such as integral cross sections. A qualitative discussion in terms of broad and narrow resonances is given to justify the disappearance of the oscillations in our case study.

\section{References}

1. D. M. Neumark, A. M. Wodtke, G. N. Robinson, C. C. Hayden, K. Shobatake, R. K. Sparks, T. P. Schafer and Y. T. Lee, J. Chem. Phys. 82, 3067 (1985). A. H. Zewail, Science 242, 1645 (1988). R. B. Metz, A. Weaver, S. E. Bradforth, T. N. Kitsopoulos and D. M. Neumark, J. Phys. Chem. 94, 1377 (1990).

2. Faraday Discuss 91, "Structure and Dynamics of Reactive Transition States" (in press).

3. J. M. Launay and M. LeDourneuf, Chem. Phys. Lett. 1969473 (1990).

4. R. D. Levine and S. Wu, Chem. Phys. Lett. 11, 557 (1971).

5. E. Pollak and M. S. Child, Chem. Phys. 60, 23 (1981).

6. W. H. Miller and J. Z. H. Zang, J. Phys. Chem. 95, 12 (1991).

7. A. Kuppermann, J. A. Kaye and J. P. Dwyer, Chem. Phys. Lett. 74, 257 (1980). G. Hauke, J. Manz and J. Römelt, J. Chem. Phys. 73, 5040 (1980).

8. J. M. Bowman and K. T. Lee, J. Chem. Phys. 72, 5071 (1980). V. Khare, D. J. Kouri and M. Baer, J. Chem. Phys. 71, 1188(1979). H. Nakamura, A. Ohsaki and M. Baer, J. Phys. Chem. 90, 6176(1986).

9. J. M. Launay and M. LeDourneuf, J. Phys. B 15, L455 (1982).

10. X. Giménez, J. M. Lucas and A. Aguilar, J. Mol. Struct (THEOCHEM) 166, 403 (1988). A. Aguilar, M. Albertí, X. Giménez, M. González, J. M. Lucas, R. Sayós and A. Solé, in "Quantum Chemistry: Basic Aspects, Actual Trends.” R. Carbó (Ed.). Elsevier, Amsterdam, 1989. X. Giménez, J. M. Lucas and A. Aguilar, Chem. Phys. 136, 115 (1989).

11. J. R. Taylor, "Scattering Theory." John Wiley \& Sons, New York, 1972.

12. V. Aquilanti, in: “The Theory of Chemical Reaction Dynamics." D. C. Clary (Ed.). Reidel, Dordrecht, 1986.

13. A. Laganà, A. Aguilar, X. Giménez and J. M. Lucas, J. Chem. Phys. 95, 2218 (1991). A. Laganà, A. Aguilar, X. Giménez and J. M. Lucas, Faraday Discuss 91, (in press). 